\title{
ACOLHIMENTO NO PROCESSO DE TRABALHO DO ENFERMEIRO DA ATENÇÃO BÁSICA: ESTUDO QUALITATIVO
}

\section{USER EMBRACEMENT IN THE WORK PROCESS OF NURSES OF PRIMARY CARE: STUDY QUALITATIVE}

\author{
Maria de Lourdes Silva Marques Ferreira ${ }^{1}$
}

\section{RESUMO}

Objetivo: Analisar a percepção de enfermeiros da atenção básica de saúde sobre acolhimento no processo de trabaIho. Métodos: Pesquisa qualitativa, realizada com 21 enfermeiras de município do interior paulista, Brasil. A análise dos dados pautou-se na vertente fenomenológica, modalidade estrutura do fenômeno. Resultados: O acolhimento no processo de trabalho revelou-se como sistematização do trabalho, escuta das necessidades do usuário e estabelecimento de vínculo, organização da demanda para que o usuário consiga agendamento à consulta médica, como demonstração de interesse pelas necessidades do usuário, além de atribuir valor ao mesmo por se tratar do cenário de atenção básica. Estes dados mostram que é preciso se responsabilizar pelo usuário desde o momento que ele entra na unidade, entretanto, ainda há profissionais que consideram a importância do acolhimento como sinônimo de recepção e assim, referem-se a porta de entrada. $\mathrm{O}$ acolhimento também identifica as vulnerabilidades que podem comprometer a saúde e a vida das pessoas. Relaciona-se ainda à organização do acesso à consulta médica, em conformidade com o desejo da pessoa. Considerações Finais: Observam-se esforços dos diferentes atores para incorporar o acolhimento na mudança do modelo assistencial. A proposta do acolhimento surge para auxiliar a mudança do modelo assistencial médico centrado com ações curativas para estabelecer relações efetivas e desenvolver a autonomia do usuário, e com isso reafirmar a busca do cuidado integral, para fazer da Atenção Básica à Saúde um caminho melhor para a assistência à saúde.

DESCRITORES: Acolhimento; Atenção Primária a Saúde; Sistema Único de Saúde.

\section{ABSTRACT}

Objective: To analyze the perception of nurses in primary health care at the reception in the work process. Methods: Qualitative research, conducted with 21 nurses São Paulo, Brazil. Data analysis was oriented phenomenological structure, the way the phenomenon. Results: The host in the work process proved to be a systematic work, listen to user needs, establish a relationship, organize the search so that the user can schedule medical appointments, as a demonstration of interest by the user needs, and assign value to it because it is the context of primary care. These data show that it is necessary to take responsibility for the user from the moment he enters the unit, however, there are still professionals who consider the importance of the host as a synonym of reception and thus, refer to the gateway. The host also identifies vulnerabilities that can compromise people's health and life. It is also related to the organization of access to medical consultation, according to the desire of the person. Final Thoughts: It was observed that there are efforts to incorporate the host as a proposal to help change the model of care and thus reaffirm the search for integral care, to make Basic Health Care a better way for health care.

DESCRIPTORS: User Embracement; Primary Health Care; Unified Health System

\section{RESUMEN}

Objetivo: Analizar la percepción de enfermeras en la atención primaria de salud en el cuidado mientras que el proceso de trabajo. Métodos: La investigación cualitativa, realizada con 21 enfermeras Sao Paulo, Brazil. El análisis de datos se basa en la estructura fenomenológica, así fenómeno localizado. Resultados: El cuidado en el proceso de trabajo ha demostrado ser un trabajo sistemático, le permite escuchar las necesidades del usuario y establecer una relación, organizar la demanda de manera que el usuario puede programar citas, como una muestra de interés en las necesidades del usuario, y asigna el valor que, como es el área de la atención primaria. Estos datos muestran que debemos ser responsables para el usuario desde el momento en que entra en la unidad, sin embargo, todavía hay profesionales que tengan en cuenta la importancia de la acogida como sinónimo de recepción y por lo tanto

\footnotetext{
Professora Assistente Doutora do Departamento de Enfermagem da Faculdade de Medicina de Botucatu
} 
se refieren a la pasarela. El anfitrión también identifica las vulnerabilidades que pueden poner en peligro la salud y la vida de las personas. Se refiere también a la organización del acceso a la consulta médica de acuerdo con los deseos de la persona. Consideraciones finales: Se observa a partir de diferentes actores esfuerzos para incorporar un cambio en la forma de atención en el modelo de atención de la salud. La propuesta del huésped parece ayudar a cambiar el modelo médico de atención centrada en las acciones curativas para establecer relaciones eficaces y desarrollar más autonomía, y así reafirmar la búsqueda de atención integral, para que la atención primaria de salud una mejor manera de cuidado de la salud.

DESCRIPTORES: Acogimiento; Atención Primaria de Salud; Sistema Unico de Salud.

\section{INTRODUÇÃO}

A atenção básica, como um dos eixos estruturantes do SUS, vive na atualidade um momento especial ao ser assumida como uma das prioridades do Ministério da Saúde. Nesse sentido, a Política Nacional de Humanização deixa de ser apenas um programa a ser aplicado aos serviços de saúde, mas uma política que opera de forma transversal em toda a rede. Para isso, o acesso e o acolhimento são concebidos como princípios do SUS e favorecem a obtenção de melhores resultados nas intervenções em saúde. Possibilitam a continuidade do cuidado em qualquer nível de atenção, por serem elementos essenciais à assistência que visam à qualificação do cuidado prestado. ${ }^{1}$

Ainda que a necessidade de estabelecer vínculos com a clientela assistida seja condição fundamental para concretizar o acolhimento, observa-se que o mesmo tem assumido diferentes formatos e vieses, que dificultam sua efetivação no âmbito da atenção primaria à saúde. Nessa perspectiva, considera-se que o acolhimento tem que avançar e sair da posição das relações rotineiras de trabalho (assistencialismo) meramente burocrático para incorporar-se no sistema de atendimento como forma facilitadora na promoção do bem-estar físico, mental e social do usuário do sistema público de saúde. ${ }^{2}$

Em um contexto de mudanças, as novas propostas de cuidado são necessárias, profundas e desafiadoras. Implicam na transformação de postura dos profissionais da equipe de saúde, tem-se como destaque o papel do enfermeiro como um profissional que tem sob sua responsabilidade outros integrantes da equipe de enfermagem, além de muitas vezes assumir a gestão do serviço.

Dada a relevância do papel do enfermeiro na atenção primária, as instituições formadoras têm se preocupado em desenvolver as competências inerentes a esse trabalho. Em análise da percepção do enfermeiro sobre o trabalho na Estratégia Saúde da Família, mostrou que a forma de conceber o trabalho é permeada por avanços, limites e contradições inerentes ao momento de transição, sendo considerado que há necessidade de aproximação reflexiva entre o trabalho como concebido e o cotidiano, com vistas à constante reconstrução das práticas. ${ }^{3}$

Frente à relevância dada ao acolhimento na construção de um modelo de atenção à saúde e a relevância do processo de trabalho da enfermagem, em permanente construção, tem-se como questionamento: como os enfermeiros estão incorporando o acolhimento no seu processo de trabalho? O objetivo deste estudo foi analisar a percepção de enfermeiros da atenção básica de saúde sobre acolhimento no seu processo de trabalho.

\section{MÉTODO}

Trata-se de estudo qualitativo, na qual se utilizou o método fenomenológico. A coleta de dados foi no período de fevereiro a julho de 2013 com enfermeiros da atenção primária à saúde de oito municípios localizados na região noroeste do estado de São Paulo. A rede básica de saúde, para os municípios em estudo, compõe-se de 30 unidades de Atenção Primária à Saúde, sendo sete na modalidade da Estratégia de Saúde da Família (ESF), 23 tradicionais, e destas, 10 incorporam o Programa de Agente Comunitário de Saúde (PACS).

$O$ instrumento de coleta de dados contou com dados de caracterização (função exercida, especialização na área e características da unidade) e uma questão norteadora: como você percebe o acolhimento como processo de trabalho?

A amostra constituiu-se por 21 enfermeiras, definida pelo critério da saturação teórica, que leva à suspensão da inclusão de participantes quando os dados passam a apresentar, na avaliação do pesquisador, certa redundância ou repetição. ${ }^{5}$ As entrevistas foram transcritas e codificadas, resguardando a identidade dos participantes.

Para a análise dos dados, inicialmente, foram realizadas repetidas leituras atentivas dos discursos e na sequência, identificaram-se as unidades de significado, para caminhar em direção à intersubjetividade e desta- 
car os dados significativos da experiência vivida para a compreensão do fenômeno pesquisado. A extração da essencialidade da percepção sobre acolhimento enquanto processo de trabalho em saúde e a identificação das unidades de significados levou a uma redução na busca da "percepção de acolhimento para os enfermeiros". Após, estabeleceu-se a redução fenomenológica, cujas expressões cotidianas dos sujeitos da pesquisa foram transformadas na linguagem do pesquisador. ${ }^{5}$

Em seguida, foi realizada a etapa de compreensão ou interpretação, que se iniciou pela análise ideográfica, de forma a evidenciar a estrutura do fenômeno situado: ser enfermeiro e sua percepção sobre acolhimento dos pacientes atendidos. Na busca das unidades de significados, os discursos foram agrupados de acordo com categoria comum, conforme as várias significações sobre acolhimento. Após, procedeu-se a análise nomotética, que se constitui na busca de generalidade para apreender os aspectos mais comuns dos depoimentos e a essencialidade do fenômeno para desvelá-lo. ${ }^{5}$

O estudo cumpriu com os requisitos formais contidos nos padrões nacionais e internacionais de regulação de pesquisa envolvendo seres humanos.

\section{RESULTADOS}

A totalidade dos participantes do estudo foi do sexo feminino, a maioria casada e com idade entre 20 e 45 anos. Prevaleceu a ocupação de função de responsabilidade técnica de serviços de enfermagem, além da coordenação do PACS. As participantes atuavam em unidades básicas tradicionais com ou sem o PACS, bem como na ESF.

Em relação à formação complementar, a maioria das entrevistadas cursou especialização nas áreas de cuidados a pacientes críticos, urgência e emergência, enfermagem do trabalho e obstetrícia.

$\mathrm{Na}$ percepção das enfermeiras, o acolhimento revelou-se em distintos sentidos, sendo ele inerente à sistematização do trabalho, como escuta das necessidades do usuário e estabelecimento de vínculo, organização da demanda para que o usuário consiga agendamento à consulta médica, demonstração de interesse pelas necessidades do usuário, além de atribuir valor ao mesmo por se tratar do cenário de atenção básica.

\section{Sistematização da assistência}

As enfermeiras revelaram que o acolhimento é forma de sistematizar a assistência, fornecendo o suporte necessário ao usuário e sendo resolutiva, o que por certo, cumpre com um importante papel quando se trata do atendimento na atenção básica. Inclui ainda, se responsabilizar pelo usuário desde o momento que ele entra na unidade.

Acolhimento seria, para mim, a forma sistematizada de você atender o paciente fornecendo suporte necessário (Dep 7).

Se o enfermeiro sistematiza a assistência de enfermagem na unidade, o acolhimento passa a ser uma etapa importante e resolutiva (Dep 10).

O enfermeiro tem que ser responsável pelo plano de cuidado, de tratamento, enfim desde a hora que entra na unidade, isso pra mim é acolhimento (Dep 5).

\section{Escuta das necessidades do usuário e vínculo}

As enfermeiras perceberam o acolhimento como forma de escuta e vínculo do usuário que procura a unidade de saúde, sendo essa uma condição fundamental no atendimento das necessidades do usuário. Para tanto, além de desenvolver as ações possíveis dentro da atenção básica, o profissional deve contar com uma rede de atenção para dar vazão às condições específicas, por meio do encaminhamento. Neste sentido, o acolhimento também identifica as vulnerabilidades que podem comprometer a saúde e a vida das pessoas.

[...] Acolhimento é acolher, ouvir, procurar resolver ou encaminhar para que tenha as suas necessidades satisfeitas, objetivo este que procurou a unidade de saúde [...] (Dep18).

Por exemplo, o grupo de gestante [...], ela é acoIhida, porém se ela apresentar alteração que foge do pré-natal normal, ela deve ser encaminhada, não deixa a gestante vulnerável a alterações que possam comprometer a saúde (Dep 20).

Para o acolhimento é preciso ter vínculo com o paciente... (Dep 19)

No que ser refere à escuta, as entrevistadas também colocam que o acolhimento depende do interesse demonstrado para o outro e da comunicação, que é preciso ir para além da escuta, por meio da tomada de providências e orientações, conforme segue:

Se você tiver uma linguagem autoritária, como vai fazer acolhimento... (DEP 8).

\section{Organização do acesso para a consulta médica}

As enfermeiras desvelaram ainda que o acolhimento se relaciona à organização do acesso à consulta médica, em conformidade com o desejo da pessoa. Revelam, no entanto, que há valorização do modelo 
médico centrado, ao indicar como bom o acolhimento que proporciona atendimento com o médico de preferência do usuário.

A gente aqui tem um acolhimento bom. Aqui é assim, a pessoa chega à consulta, é agendada na janela. Não tem nada de senha, a pessoa chega aqui no fichário. A gente encaixa com o médico que o paciente deseja (Dep 12).

Acolhimento para mim é agendamento correto, ter horários para agendar, ter médicos para atender, acoIher é oferecer condições para atendimento do paciente, incluindo espaço físico e profissionais (Dep 14).

\section{A atenção básica como porta de entrada e a valorização do acolhimento}

As enfermeiras quando questionadas, reconhecem a importância do acolhimento na atenção básica, por ser a porta de entrada do sistema saúde, ou seja, é dela que depende todo o fluxo do atendimento necessário ao usuário.

Se é porta de entrada ao sistema de saúde, o acolhimento é importantíssimo, o usuário vai entrar neste sistema para dar início a resolução de seus problemas ( Dep 4).

As enfermeiras também compreendem como ação pontual, que deve ocorrer na recepção dos serviços básicos de saúde.

Acolhimento para mim é a pessoa chegar a recepção, ser bem acolhida. As pessoas sabem questionar, perguntar porque ela está ali (Dep 1).

\section{Acolhimento como demonstração de interesse}

As enfermeiras acreditam que o acolhimento deve ocorrer desde a recepção, por meio da demonstração de interesse pelas necessidades que o usuário.

[...] tudo depende do perfil do funcionário que eu tenho lá na frente [...] (Dep 16).

Acolher? É ser receptivo, é proteger, é se interessar pelo outro. Para trabalhar na saúde tem que ter o perfil de acolher, receber, agendar, se interessar[...] (Dep 15)

\section{DISCUSSÃO}

Esquematicamente, o processo de acolhimento pode ser definido em três etapas: inicialmente as necessidades de saúde são acolhidas em relações de diálogo e vínculo; em seguida busca-se a compreensão e significação de tais necessidades, correlacionando-as ao contexto socioeconômico, cultural e subjetivo do usuário. Posteriormente busca-se a corresponsabiliza- ção entre profissionais e usuários pelas estratégias de intervenção estimulando a autonomia destes últimos na compreensão e controle de seu quadro de saúde. ${ }^{6}$

Assim, compreendemos que ressignificar o trabaIho com elementos novos para que haja uma interação entre trabalhadores e usuários do sistema de saúde é uma forma de realizar acolhimento no processo de trabalho do dia a dia.

A proposta do acolhimento, articulada com outras propostas de mudança no processo de trabalho e gestão dos serviços (cogestão, ambiência, clínica ampliada, programa de formação em saúde do trabalhador, direitos dos usuários e ações coletivas) é um dos recursos importantes para a humanização dos serviços de saúde. Contudo, ainda é preciso maior comprometimento dos gestores em saúde, de um sistema público mais organizado, de uma rede assistencial mais organizada que garanta uma assistência que contemple o acolhimento no processo de trabalho em saúde. ${ }^{7}$

O acolhimento é uma intervenção que objetiva a escuta do universo do paciente. Trata-se de uma escuta qualificada com potencial terapêutico, e contribui para a melhoria da atenção centrada na pessoa. Neste contexto, a escuta ativa e comprometida é fundamental para a concretização da política do acolhimento nos serviços de saúde, assim como, eliminar a atitude de muitos profissionais de "coisificar" as pessoas. ${ }^{8}$

Nesta escuta, buscar a avaliação da demanda, identificar riscos e vulnerabilidades e, se comprometer em dar uma resposta. Essa postura permite que a assistência deixe de ser caracterizada por ações pontuais e isoladas e se multiplique em inúmeras outras ações capazes de reformular todo o processo de produção da saúde. ${ }^{6}$

A sinalização do acolhimento como espaço de escuta indica que esse, como um conceito-ferramenta, tem potência de produzir efeitos terapêuticos em sujeitos que se encontram em sofrimento psíquico e que o ato de acolher torna-se um ato de cuidado. Configura-se, ainda, em saber quem vai receber o usuário que chega; como avaliar o risco e a vulnerabilidade desse usuário; o que fazer de imediato; quando encaminhar/agendar uma consulta médica; como organizar a agenda dos profissionais; que outras ofertas de cuidado (além da consulta) podem ser necessárias. ${ }^{9}$

A medicalização é um processo sociocultural que transforma em necessidades médicas as vivências que eram administradas no próprio ambiente familiar. Ela acentua a realização de procedimentos profissionalizados, diagnósticos e terapêuticos, que são muitas vezes 
desnecessários. A atenção básica em saúde, se propõe a superar essa tradição da medicalização, substituindo-a pela promoção da saúde. ${ }^{10}$ As ações de acolhimento recebem influência política, econômica, social e histórica, e, portanto, não é responsabilidade exclusiva dos usuários e trabalhadores da saúde.

Não se trata de ação isolada, como uma entrevista individual e privativa de determinado profissional, mas sim como um processo coordenado e organizado. E que, deva ser capaz de incluir os usuários nos serviços e, ao mesmo tempo, potencializar os profissionais de saúde e gestores na construção de espaços democráticos, éticos e reflexivos para a construção de um novo modelo assistencial. ${ }^{11}$

Modelo este, que inclua o acolhimento no processo de trabalho, com relações de modo humanizado, na perspectiva do desenvolvimento da autonomia e sua efetivação estará relacionada com a utilização dos recursos disponíveis que sejam adequados para a resolução dos problemas dos usuários. Acolher do ponto de vista físico mostrou-se deficiente, necessitando de ampliação e implementação das condições existentes, representadas para os usuários especialmente pela simples disponibilidade de uma estrutura física mais adequada às necessidades do binômio profissional/paciente. ${ }^{12}$ Implica ainda, em não se restringir à noção tradicional de triagem, sendo percebido, dentro dos processos de trabalho, como ação em saúde e espaço de escuta.

A implantação de acolhimento da demanda espontânea "pede" e provoca mudanças nos modos de organização das equipes, nas relações entre os trabalhadores e nos modos de cuidar. Para acolher a demanda espontânea com equidade e qualidade, não basta distribuir senhas em número limitado (fazendo com que os usuários formem filas na madrugada), nem é possível (nem necessário) encaminhar todas as pessoas ao médico (o acolhimento não deve se restringir a uma triagem para atendimento médico).

Acolher implica em algumas considerações a serem pensadas, como, a ambiência do local e a garantia de atendimento, acesso aos serviços e escuta às necessidades. A ambiência é o tipo de tratamento ofertado com relação ao espaço físico, social, profissional e às relações que proporcionam atenção acolhedora, resolutiva e humana. A garantia de atendimento é um direito do usuário para que receba todas as ações prescritas, e que estas estejam acessíveis. Nesse sentido, é necessário que cada profissional escute o usuário e leve em consideração sua queixa ou seu pedido, já que eles têm significado diante da realidade enfrentada, como desvelado neste estudo.

A prática do acolhimento vem se integrando às diferentes formas de cuidado prestadas ao usuário e ao cuidador, o que contribui para que singularidades sejam compreendidas. Uma assistência integral contribui para dividirmos saberes e responsabilidades, estabelecendo que práticas de atenção à saúde sejam criativas e singulares contribuindo para a integralidade e resolubilidade do cuidado. ${ }^{13}$

Acolher configura-se num compromisso em oferecer ao paciente todos os serviços disponíveis para auxiliá-lo na solução de sua queixa, porém não significa que a instituição terá recursos para todas as demandas apresentadas pelos usuários, cabe ao profissional, a partir do acolhimento, saber quem poderá se beneficiar com os serviços oferecidos e quem precisa ser encaminhado para outras unidades de saúde.

Para além de uma concepção do acolhimento como ação técnico-assistencial, acolher necessita de ações mais diversificadas e pouco ritualizadas com profissionais mais flexíveis e versáteis. Neste sentido, quanto mais as pessoas trabalharem juntas, mais aberto e acessível o serviço estará a todos os tipos de demanda, aumentando a possibilidade de imersão da equipe no mundo sociocultural de sua área de abrangência, de troca de saberes pessoais e profissionais. ${ }^{14}$

No entanto, "acolher" não significa a completa resolução das demandas apresentadas pelos usuários, mas compete aos trabalhadores de saúde, conhecer a história de vida dos usuários, a fim de favorecer escoIhas e caminhos com comprometimento, segurança e esperança de uma resposta positiva ao seu perfil atual. ${ }^{15}$

As ações realizadas na porta de entrada só ganham sentido se o acolhimento for entendido como uma passagem para os processos de produção de saúde. ${ }^{5}$

Neste sentido, observam-se fragilidades neste processo, quando há o distanciamento entre o significado do acolhimento e os modos de acolher existentes na prática. Em seu significado, o acolhimento é compreendido como método de escuta atenta e qualificada, e o modo de acolher são as estratégias para que o acolhimento se efetive. O grupo pode ser uma dessas estratégias, pois traz benefícios emocionais e sociais e o acolhimento coletivo pode possuir uma função terapêutica, pela relação estabelecida entre os participantes na discussão de seus problemas, auxiliando as relações pessoais e interpessoais, por meio do diálogo. Há troca de experiências, as quais possam auxiliar na sua reabilitação e/ 
ou na convivência com os demais. ${ }^{16}$

Nesta troca de experiências, dividem saberes, responsabilidades e compromisso, possam estabelecer uma nova prática que convida o repensar ético-político do cotidiano e fortalece a prática do diálogo como forma de apoiar e estimular a criatividade e a singularidade presentes nos serviços de saúde, enquanto prática de humanização. ${ }^{17} \mathrm{O}$ acolhimento altera o processo de trabalho e, consequentemente, a gestão dos serviços de saúde, na qual os profissionais assumem compromissos com as práticas transformadoras. É por meio do acolhimento que ocorre a troca de conhecimentos, valores e crenças, e principalmente proporciona a equipe de enfermagem o interagir com a comunidade, e viabiliza a saúde e a qualidade de vida do usuário. ${ }^{18}$

Enquanto postura e prática nas ações de atenção e gestão nas unidades de saúde favorece a construção de uma relação de confiança e compromisso dos usuários com as equipes e os serviços, contribui para a promoção da cultura de solidariedade e para a legitimação do sistema público de saúde. Favorece ainda, a possibilidade de avanços na aliança entre usuários, trabalhadores e gestores da saúde em defesa do SUS como uma política pública essencial para a população brasileira. ${ }^{19}$

\section{CONSIDERAÇÕES FINAIS}

Os resultados do estudo revelaram que, em parte, os enfermeiros compreendem o significado do acoIhimento e procuram inserir os princípios do mesmo no processo de trabalho, algumas vezes compreendem que acolher significa o próprio processo de trabalho. Por outo lado, ainda existe visão restrita do significado do acolhimento, o que por certo dificulta a sua implementação. Frente a isso, depreende-se que há necessidade da inserção de abordagens teóricas e práticas sobre o acolhimento no cotidiano da formação dos enfermeiros, bem como de capacitação dos profissionais, por meio da educação permanente, para que corroborem com a realização de um acolhimento como processo coordenado e organizado para uma assistência humanizada.

A proposta do acolhimento surge para auxiliar a mudança do modelo assistencial médico centrado com ações curativas para estabelecer relações efetivas e desenvolver a autonomia do usuário, e com isso reafirmar a busca do cuidado integral, para fazer da Atenção Básica à Saúde um caminho melhor para a assistência à saúde.

\section{REFERÊNCIAS}

1. Corrêa ACP, Ferreira F, Cruz GSP, Pedrosa ICF. Acesso a serviços de saúde: olhar de usuários de uma unidade de saúde da família. Rev Gaúcha Enferm. 2011; 32(3):451-7.

2. Mitre SM, Andrade EIG, Cotta RMM. Avanços e desafios do acolhimento na operacionalização e qualificação do Sistema Único de Saúde na Atenção Primária: um resgate da produção bibliográfica do Brasil. Ciência \& Saúde Coletiva 2012; 17(8):2071-85.

3. Souza MG, Mandu ENT, Elias AN. Percepções de enfermeiros sobre seu trabalho na Estratégia de Saúde da Família. Texto Contexto Enferm 2013 Jul-Set; 22(3):772-9.

4. Holloway I, Wheller S. Phenomenology. In: Holloway I, Wheller S. Organizadores. Qualitative research in Nursing and Healthcare. Third Edition. Oxford: John \& Son; 2013. p 213-31.

5. Ministério da Saúde (BR). Secretaria de Atenção à Saúde. Departamento de Atenção Básica. Acolhimento à demanda espontânea. Brasília (DF): Ministério da Saúde; 2011.

6. Barra SAR. O acolhimento no processo de trabalho em saúde. Ser. Soc. Rev. 2011; 13(2):119-42.

7. Oliveira DA, Guimarães JP. A Importância do acoIhimento com classificação de risco nos serviços de emergência Caderno Saúde e Desenvolvimento 2013; 2(2):25-44

8. Maynart WHC, Albuquerque MCS, Brêda MZ, Jorge JS. A escuta qualificada e o acolhimento na atenção psicossocial Acta Paul Enferm. 2014; 27(4):300-3.

9. Scheibel A, Ferreira LH. Acolhimento no caps: reflexões acerca da assistência em saúde mental. Revista Baiana de Saúde Pública. 2011; 35(4):966-83.

10. Garuzi M, Achitti MCO, Sato CA, Rocha SA, Spagnuolo RS. Acolhimento na Estratégia Saúde da Família: revisão integrativa. Rev Panam Salud Publica 2014; 35(2):144-9.

11. Mitre SM, Andrade EIG, Cotta RMM. Avanços e desafios do acolhimento na operacionalização e qualificação do Sistema Único de Saúde na Atenção Primária: um resgate da produção bibliográfica do Brasil. Ciência \& Saúde Coletiva 2012; 17(8):2071-85. 
12. Medeiros FA, Araújo-Souza GC, Barbosa AA, Costa ICC. Acolhimento em uma Unidade Básica de Saúde: a satisfação do usuário em foco. Mendes ACG et al. Ciência \& Saúde Coletiva 2012; 17(11):2903-12.

13. Velasco K, Rivas LAF, Guazina FMN. Acolhimento e escuta como prática de trabalho do psicólogo no contexto hospitalar Disciplinarum Scientia. Série: Ciências Humanas 2012; 13(2):243-55.

14. Scholze A S. Acolhimento com classificação de risco para a Estratégia Saúde da Família: a prática em uma unidade docente-assistencial. Rev Bras Med Fam Comunidade 2014; 9(31):219-26.

15. Oliveira JR, Albuquerque MCS, Brêda MZ, Barros LA, Lisbôa GLP. Concepções e práticas de acolhimento apresentadas pela enfermagem no contexto da atenção básica à saúde. Rev enferm UFPE on line. 2015; 9(Supl. 10):1545-55.

16. Souza APR, Crestani AH, Vieira CR, Machado FCM, Pereira LL. O grupo na fonoaudiologia: origens clínicas e na saúde coletiva. Rev CEFAC 2011, 13(1):140-51.
17. Nora CRD, Junges JR. Política de humanização na atenção básica: revisão sistemática Rev. Saúde Pública 2013; 47(6):1186-200.

18. Fonseca GGP, Lima SBS de, Parcianello MK, Bisogno SBC, Francisco Ritter F, Badke MR. Acolhimento e vínculo no processo de fazer dos agentes comunitários de saúde: Revisão Integrativa. Saúde 2013; 39(2):0922.

19. Brasil. Ministério da Saúde. Secretaria de Atenção à Saúde. Núcleo Técnico da Política Nacional de Humanização. Acolhimento nas práticas de produção de saúde / Ministério da Saúde, Secretaria de Atenção à Saúde, Núcleo Técnico da Política Nacional de Humanização. - 2. ed. 5. reimp. - Brasília: Editora do Ministério da Saúde, 2010. 44 p.: il. color. - (Série B. Textos Básicos de Saúde) 